AperTO - Archivio Istituzionale Open Access dell'Università di Torino

\title{
Analogs of squalene and oxidosqualene inhibit oxidosqualene cyclase of Trypanosoma cruzi expressed in Saccharomyces cerevisiae
}

\section{This is the author's manuscript}

Original Citation:

Availability:

This version is available http://hdl.handle.net/2318/1084

since

Terms of use:

Open Access

Anyone can freely access the full text of works made available as "Open Access". Works made available under a Creative Commons license can be used according to the terms and conditions of said license. Use of all other works requires consent of the right holder (author or publisher) if not exempted from copyright protection by the applicable law. 


\title{
Analogs of Squalene and Oxidosqualene Inhibit Oxidosqualene Cyclase of Trypanosoma cruzi Expressed in Saccharomyces cerevisiae
}

\author{
Simonetta Oliaro-Bosso, Maurizio Ceruti, Gianni Balliano, \\ Paola Milla, Flavio Rocco, and Franca Viola* \\ Dipartimento di Scienza e Tecnologia del Farmaco, Università di Torino, 10125 Turin, Italy
}

\begin{abstract}
Recently, a number of inhibitors of the enzyme oxidosqualene cyclase (OSC; EC 5.4.99.7), a key enzyme in sterol biosynthesis, were shown to inhibit in mammalian cells the multiplication of Trypanosoma cruzi, the parasite agent of Chagas' disease. The gene coding for the OSC of T. cruzi has been cloned and expressed in Saccharomyces cerevisiae. The expression in yeast cells could be a safe and easy model for studying the activity and the selectivity of the potential inhibitors of T. cruzi OSC. Using a homogenate of $S$. cerevisiae cells expressing $T$. cruzi OSC, we have tested 19 inhibitors: aza, methylidene, vinyl sulfide, and conjugated vinyl sulfide derivatives of oxidosqualene and squalene, selected as representative of different classes of substrate analog inhibitors of OSC. The $\mathrm{IC}_{50}$ values of inhibition (the compound concentration at which the enzyme is inhibited by $50 \%$ ) are compared with the values obtained using OSC of pig liver and $S$. cerevisiae. Many inhibitors of pig liver and $S$. cerevisiae OSC show comparable $\mathrm{IC}_{50}$ for $T$. cruzi OSC, but some phenylthiovinyl derivatives are 10-100 times more effective on the $T$. cruzi enzyme than on the pig or $S$. cerevisiae enzymes. The expression of proteins of pathogenic organisms in yeast seems very promising for preliminary screening of compounds that have potential therapeutic activity.

Paper no. L9864 in Lipids 40, 1257-1262 (December 2005).
\end{abstract}

Sterols are eukaryotic membrane components that are necessary to support cell growth and differentiation. Sterol biosynthesis is a well-established chemotherapeutic target in pathogenic eukaryotes, such as fungi $(1,2)$. The final product of the sterol biosynthetic pathway in fungi is the 24-alkyl sterol ergosterol. Ergosterol or similar 24-alkyl sterols are also the final products of the sterol synthetic pathway of some pathogenic protozoa including Trypanosoma species. These organisms cannot completely substitute these sterols with the cholesterol biosynthesized in host mammalian cells and need to synthesize at least some of their own distinctive sterols (3-5). This dependence on sterols suggests that sterol biosynthesis inhibitors may be useful antiprotozoal drugs.

The sterol biosynthetic pathway (Fig. 1) offers many poten-

\footnotetext{
*To whom correspondence should be addressed at Dipartimento di Scienza e Tecnologia del Farmaco, Via P. Giuria, 9-10125 Torino, Italy.

E-mail: franca.viola@unito.it

Abbreviations: $\mathrm{IC}_{50}$, inhibitor concentration reducing enzymatic activity by $50 \%$; OSC, oxidosqualene cyclase.
}

tial targets for therapeutic purposes. Many effective antifungal drugs already in use act by inhibiting different enzymes of sterol biosynthesis; the allylamine antifungal drug terbinafine inhibits squalene epoxidase (6), and azole drugs such as ketoconazole and itraconazole are inhibitors of lanosterol $\mathrm{C}_{14^{-}}$ demethylase (7). The activity of these inhibitors can be explained as a consequence either of depletion of the ergosterol or of accumulation of toxic intermediates or side products. In addition to the antifungal activity, some of these compounds

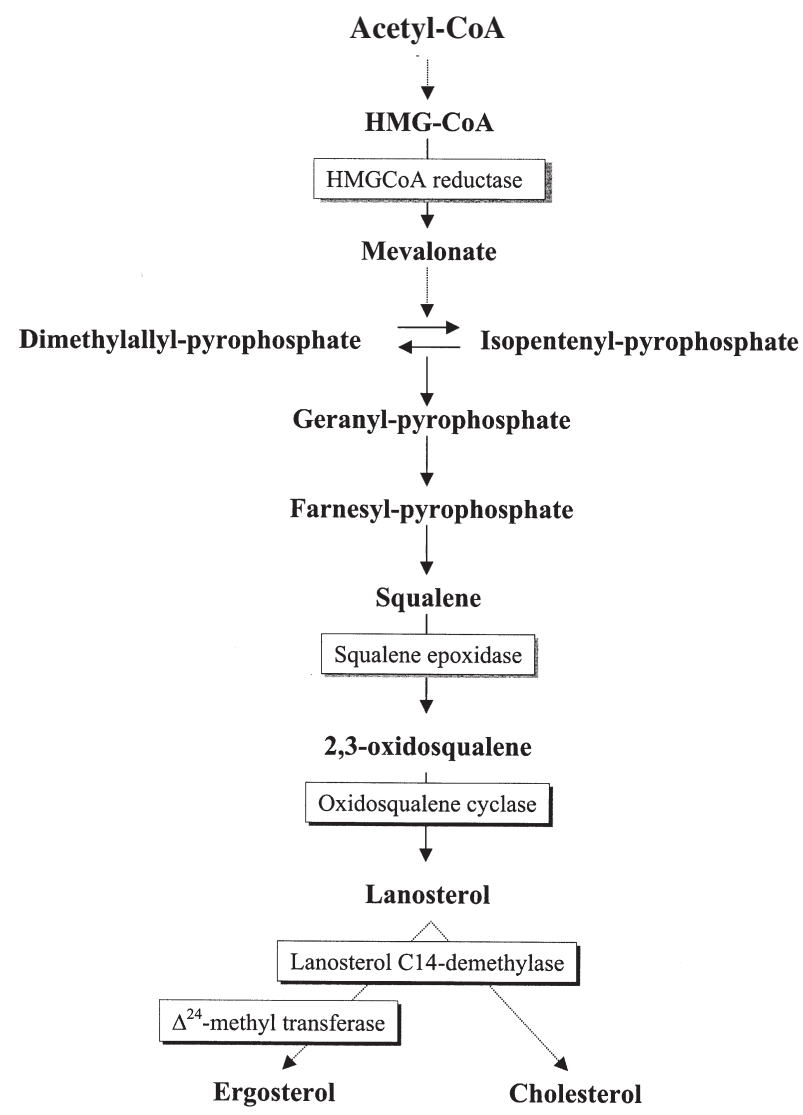

FIG. 1. Schematic representation of sterol biosynthetic pathways. The end products (ergosterol and cholesterol) are typical for fungi and animals, respectively. In the boxes are shown the enzymes cited in the text as targets for antimicrobial drugs. 
<smiles>CCCC=C1CCCC(C)(CCC=C(C)CCC=C(C)C)CCC1</smiles>

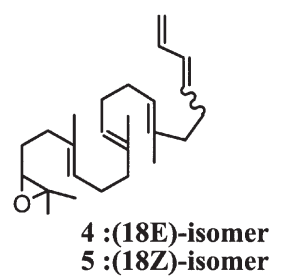<smiles>CSCCCC1=C(C)CCCC1(CCCSC)C1=CCCCC1</smiles>

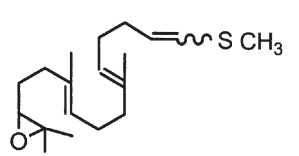

13

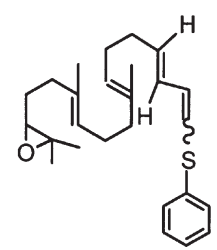

17<smiles>CCCC=C1CCCC(C)(CCC=C(C)CCC=C(C)C)CCC1</smiles>

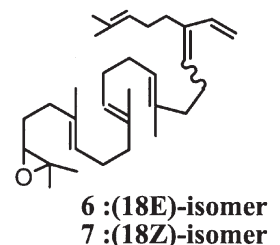

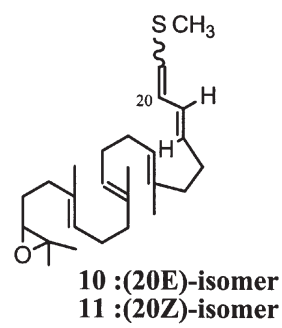<smiles>CC=CC1=CCCC=C1CCC</smiles>

14 :(16E)-isomer 15 :(16Z)-isomer

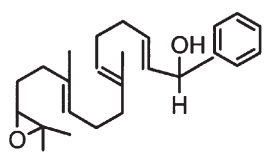

18

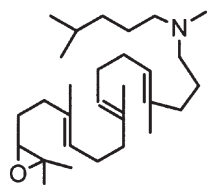

3

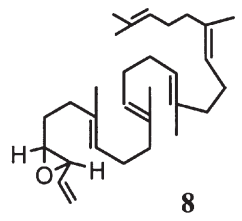

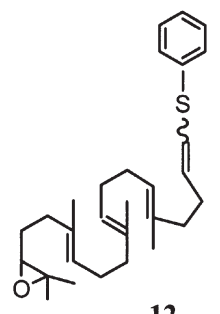

12<smiles>CC1(C)CCCCC1=CCSc1ccccc1</smiles>

16<smiles>C=CC(CC(O)CC/C=C\C(=C/C)CCC1CO1)Sc1ccccc1</smiles>

19

FIG. 2. Squalene and 2,3-oxidosqualene derivatives tested as inhibitors of Trypanosoma cruzi oxidosqualene cyclase.

were shown to inhibit the growth of Trypanosoma and other kinetoplastid parasites (8). Kinetoplastid parasites have also been shown recently to be susceptible to inhibitors of squalene synthase $(9,10)$ and oxidosqualene cyclase $(11,12)$.

The enzyme oxidosqualene cyclase (OSC; EC 5.4.99.7), which catalyzes the formation of the first cyclic precursor of sterols, is considered a good target for the inhibition of sterol biosynthesis in both humans and fungi. Many inhibitors, designed on the basis of the complex cyclization mechanism of OSC (13), are active on the enzyme and have been investigated as potential cholesterol-lowering or antifungal drugs $(1,2,13)$. The recent crystallization of human OSC as a complex with the potent inhibitor RO48-8071 opens the way to new structurebased studies of the cyclization mechanism (14).

We have designed and tested against mammalian and fungal OSC many acyclic substrate analogs modified either to mimic the high-energy carbocationic intermediates (compounds 1-3 of Fig. 2) or to bear reactive functions able to interact with the active site residues, as the methylidene (compounds 4-8), vinyl sulfide (compounds $9, \mathbf{1 2}, \mathbf{1 3}, \mathbf{1 6}$ ), conjugated vinyl sulfide (compounds 10, 11, 14, 15, 17), hydroxysulfide (compound 19), or hydroxyphenyl derivatives (compound 18) (15-20). OSC of different origins can have significantly different susceptibilities to the inhibitors, showing 
the possibility of selective inhibition $(19,20)$. Therefore, it would be worth testing the activity of these inhibitors on the OSC of the pathogen protozoon Trypanosoma cruzi, which recently has been shown to be susceptible to pyridinium ionbased inhibitors and to some umbelliferone aminoalkyl derivatives $(11,12)$. Trypanosoma cruzi, like other pathogenic protozoa, is difficult to culture for an extensive, preliminary screening of the many available, effective inhibitors of OSC. Recently, the gene coding for the OSC of T. cruzi, a lanosterol synthase, has been cloned and expressed in Saccharomyces cerevisiae (21). The enzyme expressed in the yeast cells could be a safe and easy model for a preliminary study of the activity and the selectivity of the potential inhibitors of T. cruzi OSC.

In this paper we report the activity of different types of substrate analogs as inhibitors of the T. cruzi OSC expressed in $S$. cerevisiae cells. Basically, three types of inhibitors were studied: analogs of the carbocationic intermediates, as the azasqualenes $(15,18)$; oxidosqualene derivatives bearing a conjugated methylidene group, designed as affinity-labeling inhibitors $(16,22)$; and vinyl sulfide, and conjugated vinyl sulfide derivatives of oxidosqualene, very effective inhibitors of yeast OSC (17,19,20), (Fig. 2).

\section{MATERIALS AND METHODS}

Materials, substrates, and test compounds. All the components of buffers and culture broths were obtained from SigmaAldrich (Milan, Italy) unless otherwise specified.

The substrate of OSC, 2,3-oxidosqualene, was synthesized as previously described (23). The labeled $\left[{ }^{14} \mathrm{C}\right]-(3 S) 2,3$-oxidosqualene was obtained through biological synthesis by incubating a pig liver $\mathrm{S}_{10}$ supernatant with $R, S\left[2-{ }^{14} \mathrm{C}\right]$ mevalonic acid $(55 \mathrm{mCi} / \mathrm{mmol}, 2.04 \mathrm{GBq} / \mathrm{mmol}$ ) (Amersham Pharmacia Biotech, Buckinghamshire, United Kingdom), in the presence of the OSC inhibitor U-14266A (24), as previously described (19). $\left[2-{ }^{14} \mathrm{C}\right]$ Acetate $(50 \mathrm{mCi} / \mathrm{mol})$ was obtained from Amersham Pharmacia Biotech. The synthesis of the inhibitors tested (compounds 1-19 of Fig. 2) has been described elsewhere $(16,17,19,20,23,25,26)$. The identity and purity of inhibitors was checked by TLC, ${ }^{1} \mathrm{H}$ NMR and mass spectra before testing the inhibition of T. cruzi OSC.

Strains of S. cerevisiae and culture conditions. SMY8pBJ1.21 strain, expressing the OSC of T. cruzi; SMY8pSM61.21, expressing the wild-type yeast OSC; and SMY8pSM60.21, expressing the Arabidopsis thaliana OSC obtained by transformation of lanosterol synthase mutant strain of S. cerevisiae SMY8 (MAT $\alpha$ erg $7:: H I S 3$ hem 1::TRP1 ura3-52-trpl-463 leu2-3.112 his3- -200 ade $2 \mathrm{Gal}^{+}$) were kindly provided by Professor S.P.T. Matsuda [Department of Chemistry and Biochemistry and Cell Biology, Rice University, Houston, TX $(21,27,28)]$. All strains were grown in a YPD culture broth (yeast extract 1\%, peptone $2 \%$, dextrose $2 \%)$ containing hemin $(0.013 \mathrm{mg} / \mathrm{mL})$ and ergosterol $(0.02 \mathrm{mg} / \mathrm{mL})$. Hemin had to be added to the growth medium since the SMY8 strains contains a mutation (hem1::TRP1) affecting heme biosynthesis. The presence of a heme mutant background is necessary for the viability of lanosterol synthase mutants in aerobic conditions (27). OSC expression was induced in YPG broth (yeast extract 1\%, peptone 2\%, galactose $2 \%)$ containing hemin $(0.013 \mathrm{mg} / \mathrm{mL})$. All strains were preserved in $40 \%$ glycerol at $-80^{\circ} \mathrm{C}$.

Enzymatic assays. The activity of OSC was assayed in cellfree homogenates obtained from cultures grown to late exponential phase in YPG at $30^{\circ} \mathrm{C}$. The cells were centrifuged at $3000 \times g$ for $10 \mathrm{~min}$ and the homogenates prepared by lysing the cell wall with lyticase $(2 \mathrm{mg} / \mathrm{g}$ wet cells in $1.2 \mathrm{M}$ sorbitol, $0.02 \mathrm{M} \mathrm{KH}_{2} \mathrm{PO}_{4}, \mathrm{pH} 7.4$, for $60 \mathrm{~min}$ at $30^{\circ} \mathrm{C}$ ). The spheroplasts obtained after lysis were sedimented at $3000 \times g$ for $10 \mathrm{~min}$, washed twice with $1.2 \mathrm{M}$ sorbitol, $0.02 \mathrm{M} \mathrm{KH}_{2} \mathrm{PO}_{4}$ and homogenized with a Potter device in $10 \mathrm{mM}$ MES/TRIS buffer, containing $0.2 \mathrm{mM}$ EDTA and $1 \mathrm{mM}$ PMSF, pH 6.9. Proteins in the homogenate were quantified with the Protein Assay Kit SIGMA, based on the method of Lowry, modified by Peterson (29). The homogenates could be used fresh or after storage at $-80^{\circ} \mathrm{C}$ for several months. The OSC activity was assayed by incubating the homogenate in the presence of the labeled $\left[{ }^{14} \mathrm{C}\right]-$ $(3 S)$-2,3-oxidosqualene $(1000 \mathrm{cpm})$ diluted with unlabeled $(R, S) 2,3$-oxidosqualene to a final concentration of $25 \mu \mathrm{M}$. Cold and labeled substrates and inhibitors, when present, were added as solutions in $\mathrm{CHCl}_{3}$ to test tubes in the presence of Tween- 80 $(0.2 \mathrm{mg} / \mathrm{mL}$ of final volume $)$ and Triton X-100 $(1 \mathrm{mg} / \mathrm{mL}$ of final volume). The solvent was evaporated under nitrogen. The substrate and inhibitors were dissolved in $50 \mu \mathrm{L}$ of MES/TRIS buffer, containing $0.2 \mathrm{mM}$ EDTA, pH 6.9 and the amount of homogenate necessary to obtain a $20 \%$ substrate transformation was added and diluted to a final volume of $250 \mu \mathrm{L}$ buffer. After $30 \mathrm{~min}$ of incubation at $35^{\circ} \mathrm{C}$ in plugged tubes, the enzymatic reaction was stopped by adding $1 \mathrm{~mL}$ of $\mathrm{KOH}$ in methanol $(10 \% \mathrm{wt} / \mathrm{vol})$ and heating at $80^{\circ} \mathrm{C}$ for $30 \mathrm{~min}$ in a water bath. After extracting with $2 \mathrm{~mL}$ of petroleum ether, the solvent was evaporated and the extract was dissolved in a small amount of $\mathrm{CH}_{2} \mathrm{Cl}_{2}$ and spotted on TLC plates (Alufolien Kieselgel 60F254; Merck, Darmstadt, Germany) using $n$-hexane/ethyl acetate $(85: 15, \mathrm{vol} / \mathrm{vol})$ as developing solvent. The conversion of the labeled substrate into labeled lanosterol was analyzed by a System 200 Imaging Scanner (Hewlett-Packard, Palo Alto, CA); the counts were collected for $5 \mathrm{~min}$, and the percentage of transformation was calculated by integration. For the $K_{\mathrm{m}}$ determinations different final concentrations of substrate $(1-200 \mu \mathrm{M})$ were used and the incubation times arranged to obtain percentages of transformation not exceeding $10 \%$ (to keep safely within the range of linear relationship between product formation and time of reaction). The values of the enzymatic rate obtained at the different substrate concentrations were fitted by a nonlinear regression method to the MichaelisMenten equation to calculate the $K_{\mathrm{m}}(30)$.

$\mathrm{IC}_{50}$ values (the concentration of inhibitor that reduces the enzymatic conversion by $50 \%$ ) were calculated by nonlinear regression analysis of the residual activity vs. the log of concentrations of inhibitors. The values in the table are the means of two separate experiments each in duplicate. 


\section{RESULTS}

Inhibition of T. cruzi OSC in cell-free homogenate of SMY8pBJ1.21 strain. To screen the inhibitors we used a homogenate of cells of the SMY8pBJ1.21 strain grown to late exponential phase. The T. cruzi OSC, known to partition between the cytosolic lipid bodies and the microsomes (31), was highly expressed in these cells. Detergents are needed to ensure the access of the apolar substrate and inhibitors to the enzyme in the lipid bodies and in the membranes and were used according to previous experiences $(15,16,32)$. The enzyme activity was evaluated by radiometric scanning of thin-layer chromatograms of the organic extract of incubation mixtures. In our experimental conditions (plugged test tubes with no NADPH added), the only labeled peaks detected corresponded to cold standards of 2,3-oxidosqualene and lanosterol. No label was found in the 4-desmethylsterol region.

The specific activity, expressed as nmol of lanosterol formed, was $4 \mathrm{nmol} / \mathrm{h} / \mathrm{mg}$ of proteins. The value of $K_{\mathrm{m}}$, calculated by a nonlinear regression method, was $66 \pm 13.5 \mu \mathrm{M}$.

To check the reliability and suitability of this homogenate for inhibition studies, we determined that the $K_{\mathrm{m}}$ values of the $S$. cerevisiae and A. thaliana enzymes expressed in the same host were, respectively, $30 \pm 14.2$ and $91 \pm 29.6 \mu \mathrm{M}$, in agreement with the data reported for these enzymes using different enzymatic preparations $(33,34)$. Therefore, the OSC expressed in transformed SMY8 cells have the same affinity for the substrate previously determined and seem suitable for testing inhibitors and comparing the results with those previously obtained with OSC of different origin.

All the inhibitors assayed, illustrated in Figure 2 and listed in Table 1, are substrate analogs already tested on mammalian and fungal OSC and were chosen as the more representative of different classes of compounds designed to inhibit OSC by different mechanisms. Prior to testing the inhibition of T. cruzi OSC, compound 1 was tested with the $S$. cerevisiae OSC expressed in yeast, compounds $\mathbf{1 2}$ and $\mathbf{1 6}$ were tested with pig liver enzyme. The $\mathrm{IC}_{50}$ values determined and found to be the same as those reported in References 18 and 20, respectively.

Table 1 shows the $\mathrm{IC}_{50}$ values obtained with some aza and methylidene derivatives of the substrate and with a series of vinyl and conjugated sulfide derivatives of truncated oxidosqualene. The activities are compared with those obtained previously by using detergent-solubilized microsomes prepared from pig liver and $S$. cerevisiae cells. The azasqualene derivatives are the 2-aza-2,3-dihydrosqualene $\mathbf{1}$, its $\mathrm{N}$-oxide $\mathbf{2}$, and the 19-azasqualene $\mathbf{3}(25,26)$. The OSC of $T$. cruzi and of pig liver are inhibited similarly by 2 -aza derivatives $\mathbf{1}$ and $\mathbf{2}$, but not by $\mathbf{1 9}$-aza derivative $\mathbf{3}$, which is a 30 times less effective inhibitor of $T$. cruzi enzyme. The methylidene derivatives 4, 6, 7, and 8 show comparable activities against the T. cruzi and pig liver enzyme whereas compound $\mathbf{5}$ is five times less effective on $T$. cruzi OSC. As already observed for mammalian enzyme, the isomers of inhibitor with the same configuration as the substrate are at least 10 times more effective (16). The presence of a vinyl function in position 2 as in the trans 2-
TABLE 1

Inhibition of Trypanosoma cruzi OSC Expressed in SMY8pBJ1.21 Yeast Strain by Aza, Methylidene, and Vinyl and Dienic Sulfide Derivatives of Substrate Compared with IC ${ }_{50}$ Previously Obtained with OSC from Pig Liver and Saccharomyces cerevisiae

\begin{tabular}{|c|c|c|c|}
\hline \multirow[b]{2}{*}{ Compound $^{a}$} & \multicolumn{3}{|c|}{$\mathrm{IC}_{50}(\mu \mathrm{M})^{b}$} \\
\hline & $\begin{array}{c}\text { OSC } \\
\text { T. cruzi }\end{array}$ & $\begin{array}{c}\text { OSC } \\
\text { Pig liver }\end{array}$ & $\begin{array}{c}\text { OSC } \\
\text { S. } \text { cerevisiae }^{C}\end{array}$ \\
\hline 1 & 0.8 & 0.15 & 10.0 \\
\hline 2 & 4.0 & 3.3 & 16.0 \\
\hline 3 & 50.0 & 1.7 & 35.0 \\
\hline 4 & 5.5 & 3.5 & 1.5 \\
\hline 5 & 100.0 & 20.0 & 15.0 \\
\hline 6 & 2.0 & 4.0 & 5.0 \\
\hline 7 & 0.2 & 0.4 & 1.0 \\
\hline 8 & 100.0 & 100.0 & 50.0 \\
\hline 9 & 1.7 & 1.0 & 0.05 \\
\hline 10 & $>100$ & 1.2 & 0.4 \\
\hline 11 & $>100$ & 1.0 & 0.9 \\
\hline 12 & 0.09 & 7.0 & 1.0 \\
\hline 13 & 18.0 & 5.0 & 1.5 \\
\hline 14 & 12.0 & 3.6 & 6.0 \\
\hline 15 & 10.0 & 2.6 & 2.0 \\
\hline 16 & 0.07 & 7.0 & 10.0 \\
\hline 17 & 0.6 & 20.0 & 12.0 \\
\hline 18 & 0.15 & 9.0 & $>100$ \\
\hline 19 & 0.5 & 7.5 & 40.0 \\
\hline
\end{tabular}

${ }^{a}$ For structures of these compounds see Figure 2.

$b$ Values are the means of two separate experiments, each with duplicate incubations. The maximal deviations from the mean were less than $10 \%$.

${ }^{C}$ See References 16-20. OSC, oxidosqualene cyclase; $I C_{50}$, inhibitor concentration reducing enzymatic activity by $50 \%$.

vinyl-2-oxido-1,1-bisnorsqualene $\mathbf{8}$, as already observed in pig and $S$. cerevisiae OSC, is not effective for inhibition (18).

Compounds 9-19 of Table 1 are vinyl and conjugated sulfide derivatives of truncated oxidosqualene $(17,19,20)$. The $T$. cruzi OSC seems specifically inhibited by all the phenyl derivatives tested, i.e., compounds $12, \mathbf{1 6}, \mathbf{1 7}, \mathbf{1 8}$, and 19. The $\mathrm{IC}_{50}$ values for these derivatives are in the range $70-600 \mathrm{nM}$, or 10 to 100 times lower than the $\mathrm{IC}_{50}$ found in pig liver OSC. Furthermore, the corresponding methyl derivatives $9,13,14$, and 15, which are very active in pig and $S$. cerevisiae $(19,20)$, are less effective on T. cruzi OSC. The major difference is observed with compound $\mathbf{9}$, showing, with T.cruzi, a 30 times larger $\mathrm{IC}_{50}$ than with $S$. cerevisiae. Compounds $\mathbf{1 3}$ and $\mathbf{1 6}$ were also tested with the OSC of $A$. thaliana, expressed in the yeast strain SMY8pSM60.21. The $\mathrm{IC}_{50}$ of both compounds were similar, 4.5 and $2 \mu \mathrm{M}$, respectively. $\mathrm{IC}_{50}$ of phenyl derivatives 12,16 , 17 and 19, tested with the OSC of $S$. cerevisiae expressed in a SMY8-derived strain (SMY8pSM61.21) were in complete accordance with the data shown in Table 1, obtained previously $(18,20)$ with a wild-type $S$. cerevisiae $(33)$.

\section{DISCUSSION}

We used a mutant strain of $S$. cerevisiae expressing the OSC of the pathogen protozoon $T$. cruzi to test a series of OSC inhibitors, with the aim of determining the susceptibility of this 
enzyme to different types of OSC inhibitors and possibly identifying specific inhibitors. OSC can be considered a promising target to inhibit the multiplication of pathogenic microbes, but, unlike some targets for sterol inhibition, such as $\Delta^{24}$ methyl transferase, OSC also is present in the mammalian cells. Specificity for the pathogen enzyme is consequently an important prerequisite for inhibitors.

We have tested 19 inhibitors, selected as representative of different classes of inhibitors. The azasqualene derivatives, which were designed as analogs of the high-energy carbocationic intermediates generated at $\mathrm{C}-2$ after the opening of the oxiranic ring, or at $\mathrm{C}-19$, after the closure of the fourth ring, have been well studied as inhibitors of mammalian and fungal OSC $(15,25,26)$. From the comparison of the $\mathrm{IC}_{50}$ obtained with the $T$. cruzi and with the pig and $S$. cerevisiae OSC, these compounds do not show specificity for the $T$. cruzi enzyme, probably because the mechanism of the initial step of the cyclization is very conserved in all the OSC (13). The closure of the last ring and the rearrangement are probably differently controlled in different OSC, as the 19-aza-derivative is very active only against pig liver OSC. The 29-methylidene derivatives 4-7, previously shown to be time-dependent inhibitors $(16,22)$, similarly lack specificity.

The most selective inhibition of T. cruzi OSC was found in the series of vinyl-thio-derivatives. The design of these compounds was based on the hypothesis that a partial cyclization of these compounds in the active site of the enzyme, because of the excellent properties of the sulfur in stabilizing the electron-deficient $\alpha$-carbon, owing to its good $\pi$ - and $\sigma$-donor properties, could generate carbocationic intermediates that were more stable and more able to interact strongly with nucleophilic amino acid residues. Our previous results showed that a methyl sulfide derivative, such as compound $\mathbf{9}$, is a very effective inhibitor of $S$. cerevisiae OSC and a time-dependent inhibitor of pig OSC, whereas phenyl sulfide derivatives, such as $\mathbf{1 6}, \mathbf{1 7}$, and 19, are poor inhibitors of both pig and yeast $\operatorname{OSC}(17,19,20)$. The $T$. cruzi OSC seems to be specifically inhibited by all the phenyl derivatives tested (compounds 12, 16-19). On the basis of these results, we initially speculated that the inhibition could be ascribed to specific $\pi-\pi$ interaction of the aromatic derivatives with the Tyr540 of T. cruzi (21), which in the other lanosterol synthases is substituted for a conserved threonine residue. A tyrosine is conserved at this position in cycloartenol synthase $(35,36)$, the plant enzyme converting oxidosqualene into cycloartenol, the precursor of plant sterols. The type of amino acid present in this position seems catalytically relevant for determining the final product of cyclization. Mutagenesis experiments have shown that the T/Y substitution causes a loss of accuracy in determining the final product of cyclization. A $S$. cerevisiae T384Y mutant produces substantial amounts of parkeol and lanost-24-ene-3 $\beta, 9 \alpha$-diol in addition to lanosterol (35), and the Y410T mutant of $A$. thaliana does not form cycloartenol, but rather lanosterol and 9 $\beta$-lanosta-7,24-dien-3 $\beta$-ol (36). To test the hypothesis that Tyr540 determines inhibitor specificity, we studied the inhibition of compounds $\mathbf{1 3}$ and $\mathbf{1 6}$ using a homogenate of a SMY8pSM60.21 strain expressing the OSC of A. thaliana.
In contrast to our hypothesis, both the methyl and the phenyl derivatives showed similar $\mathrm{IC}_{50}$, respectively, 4.5 and $2 \mu \mathrm{M}$. Therefore, the specificity of the aromatic derivatives for the T. cruzi OSC may result from more complex differences between the two active sites, allowing different interactions with the inhibitors and possibly relating to the mechanism of control of the rearrangement and of the formation of the final product (37).

In conclusion, our results show that the homogenate of a yeast strain expressing enzymes of pathogenic organisms can be used for a preliminary screening of compounds potentially active against eukaryotic pathogenic organisms in totally safe conditions. The aromatic vinyl-thio-derivatives of the substrate of OSC are very promising candidates for the inhibition of sterol biosynthesis in $T$. cruzi and possibly related pathogens, and could also be considered as models for the design of new inhibitors.

\section{ACKNOWLEDGMENTS}

This work was supported by the Ministero dell'Istruzione, Università e Ricerca (MIUR), Italy (ex 60\%). Thanks are due to professor Seiichi Matsuda (Rice University, Houston, Texas) for supplying the $S$. cerevisiae strains SMY8pBJ1.21, expressing the T. cruzi OSC; SMY8pSM61.21, expressing the wild-type $S$. cerevisiae OSC; and SMY8pSM60.21, expressing the A. thaliana OSC.

\section{REFERENCES}

1. Mercer, E.I. (1991) Sterol Synthesis Inhibitors: Their Current Status and Modes of Action, Lipids 26, 584-597.

2. Abe, I., and Prestwich, G.D. (1999) Squalene Epoxidase and Oxidosqualene Lanosterol Cyclase-Key Enzymes in Cholesterol Biosynthesis, in Comprehensive Natural Products Chemistry. Isoprenoid Including Carotenoids and Steroids (Cane, D., ed.), Vol. 2, pp. 267-298, Elsevier, Oxford, United Kingdom.

3. Urbina, J.A. (1997) Lipid Biosynthesis Pathways as Chemotherapeutic Targets in Kinetoplastid Parasites, Parasitology 114, S91-S99.

4. Coppens, I., and Courtoy, P.J. (1995) Exogenous and Endogenous Sources of Sterols in the Culture-Adapted Procyclic Trypomastigotes of Trypanosoma brucei, Mol. Biochem. Parasitol. 73, 179-188.

5. Contreras, L.M., Vivas, J., and Urbina, J.A. (1997) Altered Lipid Composition and Enzyme Activities of Plasma Membranes from Trypanosoma (Schizotrypanum) cruzi Epimastigotes Grown in the Presence of Sterol Biosynthesis Inhibitors, Biochem. Pharmacol. 53, 697-704.

6. Ryder, N.S., Stuetz, A., and Nussbaumer, P. (1992) Squalene Epoxidase Inhibitors: Structural Determinants for Activity and Selectivity of Allylamines and Related Compounds, in Regulation of Isopentenoid Metabolism (Nes, W.D., Parish, E.J., and Trzaskos, J.M., eds.), pp. 192-204, American Chemical Society, Washington, DC.

7. Vanden Bossche, H., Marichal, P., Coene, M.-C., Willemsens, G., Le Jeune, L., Cools, W., and Verhoeven, H. (1992) Сytochrome P450-dependent 14 $\alpha$-Demethylase: Target for Antifungal Agents and Herbicides, in Regulation of Isopentenoid Metabolism (Nes, W.D., Parish, E.J., and Trzaskos, J.M., eds.), pp. 219-230, American Chemical Society, Washington, DC.

8. Docampo, R., and Schmuñis, G.A. (1997) Sterol Biosynthesis Inhibitors: Potential Chemotherapeutics Against Chagas Disease, Parasitol. Today 13, 129-130.

9. Urbina, J.A., Concepcion, J.L., Rangel, S., Visbal, G., and Lira,

Lipids, Vol. 40, no. 12 (2005) 
R. (2002) Squalene Synthase as Chemotherapeutic Target in Trypanosoma cruzi and Leishmania mexicana, Mol. Biochem. Parasitol. 125, 35-45.

10. Orene Lorentes, S., Gomez, R., Jimenez, C., Cammerer, S., Yardley, V., de Luca-Fradley, K., Croft, S.L., Ruiz-Perez, L.M., Urbina, J., Gonzalez Pacanowska, D., et al. (2005) Biphenylquinuclidines as Inhibitors of Squalene Synthase and Growth of Parasitic Protozoa, Bioorg. Med. Chem. 13, 3519-3529.

11. Buckner, F.S., Griffin, J.H., Wilson, A.J., and Van Voorhis, W.C. (2001) Potent Anti-Trypanosoma cruzi Activities of Oxidosqualene Cyclase Inhibitors, Antimicrob. Agents Chemother. $45,1210-1215$.

12. Oliaro-Bosso, S., Viola, F., Matsuda, S., Cravotto, G., Tagliapietra, S., and Balliano, G. (2004) Umbelliferone Aminoalkyl Derivative as Inhibitors of Oxidosqualene Cyclase from Saccharomyces cerevisiae, Trypanosoma cruzi, and Pneumocystis carinii, Lipids 39, 1007-1012.

13. Abe, I., Rohmer, M., and Prestwich, G.D. (1993) Enzymatic Cyclization of Squalene and Oxidosqualene to Sterol and Triterpenes, Chem. Rev. 93, 2189-2206.

14. Thoma, R., Schulz-Gasch, T., D’Arcy, B., Benz, J., Aebi, J., Dehmlow, H., Hennig, M., Stihle, M., and Ruf, A. (2004) Insight into Steroid Scaffold Formation from the Structure of Human Oxidosqualene Cyclase, Nature 432, 118-122.

15. Viola, F., Brusa, P., Balliano, G., Ceruti, M., Boutaud, O., Schuber, F., and Cattel, L. (1995) Inhibition of 2,3-Oxidosqualene Cyclase and Sterol Biosynthesis by 10- and 19-Azasqualene Derivatives, Biochem. Pharmacol. 50, 787-796.

16. Ceruti, M., Rocco, F., Viola, F., Balliano, G., Milla, P., Arpicco, S., and Cattel, L. (1998) 29-Methylidene-2,3-oxidosqualene Derivatives as Stereospecific Mechanism-Based Inhibitors of Liver and Yeast Oxidosqualene Cyclase, J. Med. Chem. 41, 540-554.

17. Viola, F., Balliano, G., Milla, P., Cattel, L., Rocco, F., and Ceruti, M. (2000) Stereospecific Synthesis of trans-Vinyldioxidosqualene and $\beta$-Hydroxysulfide Derivatives, as Potent and Time-Dependent 2,3-Oxidosqualene Cyclase Inhibitors, Bioorg. Med. Chem. 8, 223-232.

18. Viola, F., Ceruti, M., Cattel, L., Milla, P., Poralla, K., and Balliano, G. (2000) Rationally Designed Inhibitors as Tools for Comparing the Mechanism of Squalene-Hopene Cyclase with Oxidosqualene Cyclase, Lipids 35, 297-303.

19. Ceruti, M., Balliano, G., Rocco, F., Milla, P., Arpicco, S., Cattel, L., and Viola, F. (2001) Vinyl Sulfide Derivatives of Truncated Oxidosqualene as Selective Inhibitors of Oxidosqualene and Squalene Hopene Cyclases, Lipids 36, 629-636.

20. Rocco, F., Oliaro-Bosso, S., Viola, F., Milla, P., Roma, G., Grossi, G., and Ceruti, M. (2003) Conjugated Methyl Sulfide and Phenyl Sulfide Derivatives of Oxidosqualene as Inhibitors of Oxidosqualene and Squalene-Hopene Cyclases, Lipids 38, 201-207.

21. Joubert, B.M., Buckner, F.S., and Matsuda, S.P.T. (2001) Trypanosome and Animal Lanosterol Synthases Use Different Catalytic Motifs, Org. Lett. 3, 1957-1960.

22. Xiao, X., and Prestwich, G.D. (1991) 29-Methylidene-2,3-oxidosqualene: A Potent Mechanism-Based Inactivator of Oxidosqualene Cyclase, J. Am. Chem. Soc. 113, 9673-9678.

23. Ceruti, M., Balliano, G., Viola, F., Cattel, L., Gerst, N., and Schuber, F. (1987) Synthesis and Biological Activity of Azasqualenes, bis-Azasqualenes and Derivatives, Eur. J. Med. Chem. 22, 199-208.
24. Field, R.B., Holmund, C.E., and Whittaker, N.F. (1979) The Effects of the Hypocholesteremic Compound $3 \beta$-( $\beta$-dimethylaminoethoxy)-androst-5-en-17-one on the Sterol and Steryl Ester Composition of Saccharomyces cerevisiae, Lipids 14, 741-747.

25. Duriatti, A., Bouvier-Navé, P., Benveniste, P., Schuber, F., Delprino, L., Balliano, G., and Cattel, L. (1985) In vitro Inhibition of Animal and Higher Plants 2,3-Oxidosqualene-Sterol Cyclases by 2-Aza-2,3-dihydrosqualene and Derivatives, and by Other Ammonium-Containing Molecules, Biochem. Pharmacol. 34, 2765-2777.

26. Ceruti, M., Rocco, F., Viola, F., Balliano, G., Grosa, G., Dosio, F., and Cattel, L. (1993) Synthesis and Biological Activity of 19-Azasqualene 2,3-Epoxide as Inhibitor of 2,3-Oxidosqualene Cyclase, Eur. J. Med. Chem. 28, 675-682.

27. Corey, E.J., Matsuda, S.P.T., Baker, C.H., Ting, A.Y., and Cheng, H. (1996) Molecular Cloning of a Schizosaccharomyces pombe cDNA Encoding Lanosterol Synthase and Investigation of Conserved Tryptophan Residues, Biochem. Biophys. Res. Commun. 219, 327-331.

28. Hart, E.A., Hua, L., Darr, L.B., Wilson, W.K., Pang, J., and Matsuda, S.P.T. (1999) Directed Evolution to Investigate Steric Control of Enzymatic Oxidosqualene Cyclization. An Isoleucine-to-Valine Mutation in Cycloartenol Synthase Allows Lanosterol and Parkeol Biosynthesis, J. Am. Chem. Soc. 121, 9887-9888.

29. Peterson, G.L. (1977) A Simplification of the Protein Assay Method of Lowry et al. Which Is More Generally Applicable, Anal. Biochem. 83, 346-356.

30. Duggleby, R.G. (1981) A Nonlinear Regression Program for Small Computers, Anal. Biochem. 110, 9-18.

31. Milla, P., Viola, F., Oliaro-Bosso, S., Rocco, F., Cattel, L., Joubert, B.M., LeClair, R.J., Matsuda, S.P.T., and Balliano, G. (2002) Subcellular Localization of Oxidosqualene Cyclases from Arabidopsis thaliana, Trypanosoma cruzi, and Pneumocystis carinii Expressed in Yeast, Lipids 37, 1171-1176.

32. Milla, P., Athenstaedt, K., Viola, F., Oliaro-Bosso, S., Kohlwein, S.D., Daum, G., and Balliano, G. (2002) Yeast Oxidosqualene Cyclase (Erg7p) Is a Major Component of Lipid Particles, J. Biol. Chem. 277, 2406-2412.

33. Balliano, G., Viola, F., Ceruti, M., and Cattel, L. (1988) Inhibition of Sterol Biosynthesis in Saccharomyces cerevisiae by $N, N$ Diethylazasqualene and Derivatives, Biochim. Biophys. Acta 959, 9-19.

34. Taton, M., Benveniste, P., and Rahier, A. (1992) Inhibition of 2,3-Oxidosqualene Cyclases, Biochemistry 31, 7892-7898.

35. Meyer, M.M., Segura, M.J.R., Wilson, W.K., and Matsuda, S.P.T. (2000) Oxidosqualene Cyclase Residues That Promote Formation of Cycloartenol, Lanosterol, and Parkeol, Angew. Chem. Int. 39, 4090-4092.

36. Herrera, J.B.R., Wilson, W.K., and Matsuda, S.P.T. (2000) A Tyrosine-to-Threonine Mutation Converts Cycloartenol Synthase to an Oxidosqualene Cyclase That Forms Lanosterol as Its Major Product, J. Am. Chem. Soc. 122, 6765-6766.

37. Segura, M.J.R., Lodeiro, S., Meyer, M.M., Patel, A.K., and Matsuda, S.P.T. (2002) Directed Evolution Experiments Reveal Mutation at Cycloartenol Synthase Residue His477 That Dramatically Alter Catalysis, Org. Lett. 4, 4459-4462.

[Received September 6, 2005; accepted November 16, 2005] 